\title{
International drive to illuminate delirium: A developing public health blueprint for action
}

\author{
Ara S. Khachaturian $^{1}$ ｜ Kathleen M. Hayden ${ }^{2}$ ～John W. Devlin ${ }^{3}$ ～Lee A. Fleisher \\ Sarah Lenz Lock ${ }^{5}$ | Colm Cunningham ${ }^{6}$ | Esther S. Oh ${ }^{7}$ | Tamara G. Fong,9,10 | \\ Donna Marie Fick $^{11}$ | Edward R. Marcantonio ${ }^{12}$ ｜ Vijeth lyengar ${ }^{13 \dagger}$ \\ Kenneth Rockwood $^{14}$ | George A. Kuchel ${ }^{15}$ | Roderic G. Eckenhoff ${ }^{16}$ | \\ Alasdair M.J. MacLullich ${ }^{17}$ | Richard N. Jones ${ }^{18}$ | Daniel Davis ${ }^{19}$ |
}

Patricia M. D'Antonio ${ }^{20}$ ～Keith N. Fargo ${ }^{21}$ | Marilyn S. Albert ${ }^{22}$ | Jeff D. Williamson ${ }^{23}$

Shari M. Ling ${ }^{24 \ddagger} \quad$ Joan Weiss ${ }^{25 \S} \quad$ Jason Karlawish ${ }^{26}$ ～Ronald C. Petersen ${ }^{27}$

Dan G. Blazer $^{28}$ | Zaven S. Khachaturian ${ }^{1}$ | Sharon K. Inouye ${ }^{29}$

\footnotetext{
${ }^{1}$ Campaign to Prevent Alzheimer's Disease, A Maryland-based 501(c)(3) not-for-profit corporation, Rockville, Maryland, USA

${ }^{2}$ Division of Public Health Sciences, Wake Forest University Health Sciences, Winston-Salem, North Carolina, USA

${ }^{3}$ School of Pharmacy, Northeastern University, Boston, Massachusetts, USA

${ }^{4}$ Department of Anesthesiology \& Critical Care, University of Pennsylvania Health System, Philadelphia, Pennsylvania, USA

${ }^{5}$ Global Council on Brain HealthAARP, Washington, DC, USA

${ }^{6}$ School of Biochemistry and Immunology, Trinity Biomedical Sciences Institute \& Trinity College Institute of Neuroscience, Trinity College, Dublin, Ireland

${ }^{7}$ Department of Medicine, The Johns Hopkins University School of Medicine, Baltimore, Maryland, USA

${ }^{8}$ Department of Neurology, Beth Israel Deaconess Medical Center, Boston, Massachusetts, USA

${ }^{9}$ Aging Brain Center, Marcus Institute for Aging Research, Hebrew SeniorLife, Boston, Massachusetts, USA

${ }^{10}$ Harvard Medical School, Boston, Massachusetts, USA

${ }^{11}$ Penn State College of Nursing, Center of Geriatric Nursing Excellence, University Park, Pennsylvania, USA

${ }^{12}$ Division of General Medicine, Beth Israel Deaconess Medical Center, Harvard Medical School, Boston, Massachusetts, USA

${ }^{13}$ Administration for Community Living, U.S. Department of Health and Human Services, Washington, DC, USA

${ }^{14}$ Divisions of Geriatric Medicine \& Neurology, Department of Medicine, Dalhousie University \& Nova Scotia Health Authority, Halifax, Canada

${ }^{15}$ Department of Geriatrics and Gerontology, University of Connecticut, Center on Aging, University of Connecticut, Farmington, Connecticut, USA

${ }^{16}$ Department of Anesthesiology and Critical Care, University of Pennsylvania Perelman School of Medicine, Philadelphia, Pennsylvania, USA

${ }^{17}$ Edinburgh Delirium Research Group, Geriatric Medicine Unit, University of Edinburgh, Royal Infirmary of Edinburgh, Edinburgh, UK

${ }^{18}$ Department of Psychiatry \& Human Behavior, Warren Alpert Medical School, Brown University, Butler Hospital, Providence, Rhode Island, USA

${ }^{19}$ MRC Unit for Lifelong Health \& Ageing, Population Science \& Experimental Medicine, Faculty of Pop Health Sciences, University College London, London, UK

${ }^{20}$ The Gerontological Society of America, Washington, DC, USA

${ }^{21}$ Alzheimer's Association, Chicago, Illinois, USA

${ }^{22}$ Division of Cognitive Neuroscience, Department of Neurology, Johns Hopkins University School of Medicine, Baltimore, Maryland, USA

${ }^{23}$ Gerontology and Geriatric Medicine, Winston-Salem, North Carolina, USA

${ }^{24}$ Centers for Medicare and Medicaid Services (CMS), Baltimore, Maryland, USA

${ }^{25}$ Division of Medicine and Dentistry, Bureau of Health Workforce, Health Resources and Services Administration, Rockville, Maryland, USA

${ }^{26}$ Division of Geriatrics, Department of Medicine, Ralston-Penn Center, Philadelphia, Pennsylvania, USA

${ }^{27}$ Alzheimer's Disease Research Center, Department of Neurology, Mayo Clinic, Rochester, Minnesota, USA

${ }^{28}$ Psychiatry \& Behavioral Sciences, Geriatric Behavioral HealthSchool of Medicine, Durham, North Carolina, USA

${ }^{29}$ Harvard Medical School, Beth Israel Deaconess Medical Center, Marcus Institute for Aging Research, Hebrew SeniorLife, Boston, Maryland, USA
} 
Correspondence

Ara S. Khachaturian, Campaign to Prevent Alzheimer's Disease, A Maryland-based 501(c)(3) not-for-profit corporation, 451 Hungerford Drive, \#119-355, Rockville, MD 20854, USA.

Email: ara@pad2020.org

The views expressed in this paper are those of the author and not necessarily of the authors' organizations including the U.S. Administration for Community Living/Administration on Aging or the U.S. Department of Health and Human Services.

The views expressed in this paper are those of the author and not necessarily of the author's organizations including the Centers for Medicare \& Medicaid Services or the U.S. Department of Health and Human Services.

$\S$ The views expressed in this paper are those of the author and not necessarily of the author's organizations including the Health Resources and Services Administration or the U.S. Department of Health and Human Services.

Disclosures: Kathleen M. Hayden, Zaven S. Khachaturian, and Ara S. Khachaturian are editors of Alzheimer's \& Dementia: The Journal of the Alzheimer's Association, and have recused themselves from the editorial decision to publish this manuscript; Peter J. Snyder, Senior Associate Editor, Alzheimer's \& Dementia served as the handling editor for this manuscript.

\section{1 | INTRODUCTION AND BACKGROUND}

Presently, there is a clear societal and demographic imperative to identify and deliver interventions that are effective in reducing dementia. With the high failure rate of compounds in clinical trials, and with pharmaceutical development cycles typically lasting decades, ${ }^{1}$ a hybrid person-with-dementia-centered approach that precisely integrates pharmaceutical, non-pharmaceutical, lifestyle modification, and care interventions for the individual may offer more immediate results for reducing dementia burden. One important, tangible, and overlooked strategy with this proposed approach may be through the recognition and prevention of delirium.

Delirium is an acute, often fluctuating syndrome characterized by inattention and global cognitive dysfunction, typically triggered by sudden and severe illness, surgery, hospitalization, or by medications. Commonly manifesting as either hypoactive, hyperactive, normoactive, or mixed forms, delirium is common in older adults, especially among persons with dementia. Although dementia increases the risk for delirium, there is growing evidence that suggests that delirium may also increase the risk for dementia. This negative-feedback relationship between delirium and dementia contributes to increased suffering, morbidity, mortality, and disability costs. Delirium may accelerate cognitive decline in patients with dementia. Among cognitively normal or mildly impaired individuals, delirium may unmask dementia pathology thus accelerating the progression to a dementia diagnoses and also, potentially, the loss of independence.

Currently, delirium is poorly recognized, in part because of lack of a unifying definition, a lack of education, and a lack of consistent application of clinically effective assessment tools. Although effective non-pharmacologic delirium preventive strategies exist, to date, no pharmacologic approach has been shown to be effective to either treat or prevent delirium. Randomized trials and large cohort studies demonstrate that use of bundled, multicomponent nonpharmacological interventions, across health care settings, may reduce delirium occurrence by $30 \%$ or more. $^{2}$ A key public health outcome will be to test if these results will translate into a reduction in subsequent dementia. To reduce delirium and potentially reduce the dementia burden through delirium-focused efforts, a new public health campaign is needed.
Such a campaign would seek to improve the recognition of delirium, to implement strategies to prevent delirium, to develop new ways of treating established delirium, and to initiate major research programs to better understand the biology of delirium and its relationship with dementia.

\section{2 | WHY IS A DELIRIUM PUBLIC HEALTH CAMPAIGN NEEDED?}

In our view, a global public health campaign addressing delirium prevention and treatment represents an unparalleled opportunity to make potentially a meaningful impact on the global dementia burden. Aside from the converging interests of a wide range of stakeholders, the rationale for such a global health campaign incorporates these details:

1. Delirium is common in older adults.

2. Delirium is increasingly being recognized as an important risk factor and possible trigger for many brain aging disorders that affect memory, movement, and mood.

3. Delirium is linked with acceleration of cognitive decline and it may also reveal vulnerability due to pre-existing dementia pathology in non-demented or mildly impaired individuals, reducing time to dementia diagnoses.

4. Unlike most other conditions associated with brain aging, delirium with its acute onset, sometimes fleeting occurrence, and symptom fluctuation necessitates a different approach for treatment and prevention.

Due to the recognition of delirium as a risk factor for dementia, there is currently unprecedented public health potential to significantly improve the care of delirium. There is an equally important opportunity to organize coordinated major research programs to elucidate the biological mechanisms that give rise to delirium. Thus, we have simultaneously released this call to action for public policy along with a whitepaper summarizing future research priorities for delirium. The next step to move the field of delirium forward is a broadly focused global public health campaign. 


\section{3 | WHAT ARE THE PUBLIC HEALTH OBJECTIVES FOR A GLOBAL DELIRIUM CAMPAIGN?}

This ambitious multi-stakeholder campaign seeks broad consensus for a coordinated-research, coordinated-care, and coordinated-advocacy plan to address the problem of delirium. The launch of the International Drive to Illuminate Delirium (IDID) seeks to advance and synergize the field of delirium along five pillars: diagnosis, awareness, burden, biology, and policy. The outcomes for this effort will be to improve care and patient outcome, to develop better prevention and treatment strategies, and advance our understanding of delirium risk factors.

The key objective for the campaign will be to lessen the cognitive and physical burden of delirium. This includes better understanding of the relationship between delirium and dementia. The campaign will help define prevention and treatment solutions that increase an individual's ability to improve quality of life, enhance functional independence, and reduce healthcare costs.

\section{4 | AIM OF THIS PAPER: DISCUSSION OF RESULTS OF THE IDID MEETING}

Just as the participants of the Leon Thal Symposia (LTS) 2007-2010 provided the impetus for today's historic increases in Alzheimer's research and care, the IDID meeting participants, an interdisciplinary group of clinicians and advocates across the globe, seek a similar outcome for delirium. IDID proposes the launch of a multi-year international public health campaign to advance and synergize the field of delirium along the five pillars (diagnosis, awareness, burden, biology, and policy) with the goal of improving care and developing better delirium and prevention strategies. In addition, the IDID effort seeks to establish and grow additional work groups to further refine plans, milestones, and implementation procedures among these five pillars. Finally, the IDID campaign will publish follow-up reports (that include both majority and minority opinions) in both the Journal's special topics section and the broader literature to help create awareness and comments regarding IDID's evolving roadmap. Future work group reports should address critical research gaps, spur public health awareness campaigns, and generate health policy objectives. The culmination of this work will be to spur increased research and care investment targeted to reduce delirium. Through this unified delirium public health approach, the IDID campaign seeks to reduce the burden, severity, and progression of dementia.

\section{5 | ITEM 1: DISCUSSION OF IDENTIFYING DELIRIUM}

- Detecting and Identifying the Occurrence of Delirium

Delirium while common is often preventable. If undetected, unmanaged, or managed unsuccessfully (either appropriately or inappropriately), some instances of delirium may lead to long-term cognitive decline and institutionalization. As a matter of public health policy, a key question is: How do we elevate the importance of delirium and incorporate recognition of delirium into the agenda of advocacy groups? Advocacy groups include those organizations with missions focused on aging, dementia, brain health, and consumer health issues as well as professional groups representing physicians, surgeons, nurses, rehabilitation specialists, physical therapists, occupational therapists, pharmacists, and other health professions working in/with various settings (eg, ambulatory care, acute care, anesthesiology, emergency room, and long-term care). Over the past decade, many of these groups have demonstrated a growing awareness of the importance of recognizing delirium, often ahead of mainstream medical organizations.

One of the primary goals in case identification is to operationalize the detection and identification of delirium across different care settings for both clinical and research applications. A better understanding of how delirium occurs as well as its short- and long-term sequelae remains an important challenge. Our understanding of delirium, particularly in the context of dementia, requires improved education and training that considers not only the brain, but also, how the disorder interacts with other organ systems.

Across many fields, there is a heuristic definition of delirium. The process of operationalizing the definition of delirium is complex. Poorly defined terms such as altered mental status, acute confusion, acute encephalopathy, and acute brain dysfunction are routinely used. ${ }^{3} \mathrm{~A}$ unified definition of delirium across different care settings is essential to advance the field. There are many approaches to define delirium in different settings and for various audiences. When we talk about informing the public, to what extent do we really need a uniform definition? How disruptive would a uniform definition of delirium be for clinicians and scientists? One important question to address is whether we can develop a definition that is clearly understandable to the public.

The effort to describe delirium will need to balance the definitional needs to drive and evaluate activities focused on its identification, its care, and its cure. Although we do not yet know how to best define delirium in different settings, there is a clear recognition and concern that the best is the enemy of the good. Just as in the field of Alzheimer's disease (AD), there is a worry that too much focus on developing the most accurate medical definition may likely obscure a bigger picture. Outreach to both the international medical-research community and the public with a clear message about how delirium should be described and assessed will be a critical priority for this process to be successful.

- International Standards / Harmonization Activities

A strong imperative exists for the development of a roadmap on how best to recognize delirium. An important initial step will be to establish a work group that carefully reviews the evolutionary history of the various diagnostic criteria for AD. Today, the AD field emphasizes a nomenclature that separates syndrome from etiology. This is important for the 
development of new diagnostics and therapies, as it introduces a new level of specificity needed for regulatory considerations. The "AT(N)" research framework ${ }^{4}$ defines $A D$ biologically (by amyloid plaques $[A]$, tau tangles [T] and [pending validation] markers of neurodegeneration $[\mathrm{N}]$ ) irrespective of the clinical presentation.

The research $\mathrm{AT}(\mathrm{N})$ framework outlines a new nomenclature that adds greater resolution to describe a specific form of neuropathologically defined and clinically manifested dementia. In this way, an individual could have plaques and tangles and thus $A D$ neuropathology, but still be cognitively normal. Although research for the AT(N) framework continues to be evaluated, an important facet of the model is the new expression of $A D$ as progressive failures of multiple, matrixed, and interconnected brain networks. For many brain disorders, such as dementia and delirium, the use of a systems failure model eschews the unitary etiologic factor or a single cause theory of pathogenesis. ${ }^{5}$ Instead, the systems failure approach requires the identification of controlling components of the network and an understanding how different sectors of the network are coupled and ordered. A similar system failure model for delirium building on existing theoretical models 6 should be a long-range goal of IDID, particularly as one way to avoid some of the evolutionary hurdles encountered in the AD field.

There is increasing agreement among delirium researchers of the need to operationalize the definition of delirium and to implement protocols to mitigate delirium when possible. ${ }^{7}$ One common situation where such protocols might be particularly beneficial is among individuals with pre-existing neurodegenerative pathology scheduled for elective surgery, particularly among patients who are older. Such individuals are at increased risk for post-operative delirium, and the appropriate pre-operative, intra-operative, and post-operative patient management for these individuals may differ from that delivered for someone without cognitive impairment or any neurodegenerative pathology. Therefore, the incorporation of a biological profile of delirium risk and outcomes will be important to inform prevention and treatment strategies. The presence of a uniform disease pathology has been a clear advantage in developing a definition for AD. In contrast, the possible etiologies for delirium ${ }^{8}$ are numerous. However, better understanding of how measurable vulnerabilities (such as those revealed by brain imaging or cognitive testing, shared impaired sensory risk factors including hearing and visual, quantification of frailty, or biological measures of aging) contribute to delirium risk offers opportunities to tailor treatment strategies.

\section{- Establishing international working groups for future definitions}

Multiple clinical, scientific, and care perspectives must be considered simultaneously, as new knowledge helps to recalibrate the definition of delirium over time. From the clinical perspective, a future work group should consider as a strategic objective how best to provide a broad holistic evaluation of delirium risk based on predisposing factors (including genomics), precipitating factors, and biomarkers. Relatedly, the work group should reexamine the issue of stigma as it relates to the definition of delirium. For example, in the field of dementia, the term neurocognitive disorders emerged as a less-stigmatizing term than dementia. Some suggest that there was a sensitivity to dementia as a pejorative term: Terminology matters. There is an important need to explore the possibility that the term delirium may carry a similar stigma, or it may be that it is an appropriate term that simply requires proper description and explanation.

Few basic neuroscientists identify delirium as a principal focus of their research, even though there are many studies that relate to delirium without using the term delirium. This is a problem because there is relatively little basic science oriented specifically to delirium, but there are many pathophysiologic mechanisms and pathways for delirium occurrence. There is a need to understand how the presence of different illnesses and risk factors can result in delirium. There may be a necessity, from the clinical perspective, to "lump" or to have a definition that coalesces around a symptom class or symptom cluster. However, a single syndromic definition may be detrimental to understanding the complexity of delirium's biological mechanisms, and some splitting will be necessary to understand the multiplicity of etiological routes to delirium. In the brain, multiple mechanisms interact, regulating multiple different hierarchical systems and overlapping networks to maintain optimal performance. In this way, a definition that considers the phenomenological definition of delirium, ideally tied to distinct etiologic or pathophysiologic pathways, will be important to neurobiologists and neuroscientists. Moreover, there is a need to develop an understanding of how it is possible for aging, in the form of biological processes and dementia, to both represent such robust predisposing risk factors for the subsequent development of delirium.

$A D$ provides an important historical and cautionary example of how not to proceed in managing the different clinical, scientific, and care perspectives of delirium. Since the 1980s, the criteria for AD have evolved through National Institute of Aging (NIA) and National Insittute of Neurological Disorders and Stroke (NINDS) workshops. These efforts developed first clinical then neuropathological criteria for $A D$. From the beginning, the definition and diagnosis of $A D$ was identified through neuropathological criteria. This continuing tendency to emphasize neuropathology over clinical presentation made the identification of AD more etiologically focused. Later, NIA established the Alzheimer's Disease Research Centers (ADRCs) with the goal of using AD clinical characterization in addition to the established neuropathological core. Unfortunately, the original intent of developing a large sample to enable syndromic characteristics to be clustered was never actually realized. As a result, the definition of $A D$ still remains an evolving moving target. Moreover, this continued emphasis on downstream neuropathology has at times fostered a brain centric perspective that has not always permitted a sufficient scientific emphasis on broader physiological and clinical contributors by other organ systems, including factors as diverse as blood pressure regulation, hormones, inflammatory molecules, and medications.

In addition to understanding delirium as a brain disorder, which can have multiorgan sequelae, delirium needs to have a broader view, known as the care perspective. The care perspective goes beyond addressing the biological facets of delirium to recognize the 
implications for quality of life for patients and their caregivers, recognizing that delirium causes considerable distress. It also considers the economic effects for hospital administrators and healthcare payers. The care perspective recognizes a strong imperative for widespread evaluation of delirium risk and monitoring for delirium in high-risk patients. In addition, the care perspective embraces an equally strong need to abolish the complacency of only detecting delirium, without building in essential linked processes for its prevention and effective treatment. Integration of the views from hospital administrators, payers, and state providers (eg, Centers for Medicare \& Medicaid Services [CMS], and the UK National Health Service) will be critically important.

Future work groups, possibly convened by advocacy groups such as Campaign to Prevent Alzheimer's Disease 2020 (PAD2020) and AARP, will need to carefully consider succinct descriptions of delirium as well as valid and reliable reference standards. ${ }^{7,9}$ Subsequent work groups will need to progress iteratively and circulate ideas to move this effort forward. In addition, this effort requires involvement from the wider community so that all stakeholders have visibility and voice, with the understanding that caregivers, family members, and healthcare professionals each see a different part of the problem. Future work groups should review the Administration for Community Living's (ACL's) pioneering work in implementing evidence-based interventions for family caregivers and persons with dementia in home and community-based settings.

- What are some models that should be considered to help diagnostic criteria evolve?

Given the need to manage an array of different professional needs, the heterogeneity of how delirium presents (eg, spanning both hyper-active and hypo-active episodes), and the multiple concomitant triggers for an episode of delirium, there is growing acknowledgment that a phenomenological approach, incorporating the best available neuropsychological research, will be important to consider as delirium diagnostic criteria and its overall framework is refined. ${ }^{10}$ Such an approach should allow better communication among different disciplinary interests around key constructs and major parameters.

This topic will require the formation of a special work group to evaluate several meritorious case studies. The ways in which the cardiovascular disease diagnostic realm has evolved is particularly informative for this purpose. For example, the early classification of myocardial infarction used certain biomarkers (ie, troponins) to identify a condition then-called myocardial ischemic necrosis syndrome (MINS). Although today this a relatively obscure classification, the case demonstrates how in North America the Canadians made MINS a "disease" but those in the United States did not, setting up discordant definitions in the two countries. The field will need to carefully consider what is currently known (and not known) and to what extent delirium may be a continuum or may represent elevation/declination of various biomarkers. "We want to avoid the confusion of troponin-itis."

- What are best options to foster international collaboration?
Increasing research investment is being allocated for the development and harmonization of new delirium identification and staging measures. The National Institutes of Health (NIH) investment established the Network for Investigation of Delirium: Unifying Scientists (NIDUS, deliriumnetwork.org), which was funded by an NIH R-24 Network Grant. NIDUS provides an opportunity for fostering future international collaboration. In dementia research, the WorldWide Alzheimer's Disease Neuroimaging Initiative (WW-ADNI) and the World-Wide FINGERS Studies provide two examples of how national research networks have evolved into international publicprivate partnerships. A work group should devise an implementation plan to determine how the various NIDUS units, cores, and task forces can be expanded internationally. The NIDUS Delirium Research Hub has already established a detailed catalog of delirium studies. In addition, the network's Measurement Core is harmonizing existing delirium assessment and severity measures through the creation of item banks and delirium instrument information cards describing performance characteristics, the populations examined, and subsequent validation studies. There is a mentoring task force that holds an annual delirium research Delirium Boot Camp, among other activities and a pilot award task force that solicits, reviews, and awards start-up grants. A dissemination task force aims to increase general public awareness, to develop a universal syndromic terminology (clinically and general public), and to advance scientific communication around delirium.

The development of a robust reference standard drawing from international experts and the best evidence is critically important to foster consistency in delirium studies. $5,7,10$ The development of any research standard must consider the population spectrum including diversity in the population with respect to race, ethnicity, economic standing, language, education, and geography. Similarly, because the Diagnostic \& Statistical Manual of Mental Disorders, Fifth Edition (DSM-5) diagnostic criteria for delirium failed to consider affective and psychotic symptoms and also how delirium phenomenology might be skewed by baseline dementia, the capture of other phenomenological behaviors should be considered for inclusion. The incorporation of a standardized Informant report of these behavioral symptoms, similar to the Neuropsychiatric Inventory Questionnaire (NPI-Q) used in the field of dementia, would be crucial. International work groups should decide how to harmonize efforts to capture informant history and should develop strategies to handle missing, incomplete, or unreliable reports. International harmonization on how best to describe and define delirium severity and track its various trajectories is also important. A dedicated International Working Group is needed to help operationalize standards for algorithmic development. This group should also be responsible for developing a plan for establishing and sustaining an open global library that curates and certifies these algorithms and their validation data.

\section{SUMMARY ISSUES}

- Currently, there are $>40$ tools for the identification of delirium; the heterogeneity of measurement standardization hampers progress in the field. Developing a standard definition and developing a 


\section{6 | ITEM 2: DISCUSSION ON AWARENESS}

\section{1 | Informing professionals}

\subsection{1 | Medical health care and service professionals}

- The work group should consider both a clinical diagnosis and a research diagnosis. Although there ultimately may be a neuropathological marker or an array of markers for delirium, pure clinical work in patients with delirium requires both a quick diagnosis and the rapid application of delirium-reducing interventions.

- The work group should also make efforts to propose an implementation pathway using both an immediate bedside diagnosis as well as a research diagnosis.

- The goal will be to implement methods of delirium classification and identification that can evolve over time, capture the phenomenological and neuropsychological profile of any given delirium episode of delirium, and inform future definitional/classification efforts.

- Standardization/harmonization activities will need to seek agreement on the fundamental aspects of the delirium syndrome from the start. These activities will need to balance an intrinsic desire to oversimplify approaches and instruments with the field's need to capture the complexity of delirium to facilitate significant advances in our knowledge of delirium.

- With delirium being under-detected, a work group may consider amassing existing large cohort studies (see Network for Investigation of Delirium Unifying Scientists [NIDUS] Research Hub) and clinical databases where delirium detection efforts have been successful. ${ }^{11}$ One of the main conclusions arising from the 2014 American Geriatrics Society/National Institute on Aging Bedsideto-Bench Conference: Research Agenda on Delirium in Older Adults involved recognition of the early stage of the delirium field and the need to better coordinate research efforts across the world. ${ }^{12}$

- The financial implications of delirium, including length of stay and readmission rates, should be further explored and quantified to convince clinicians and hospital administrators of the importance of addressing delirium. The work group should look for opportunities where delirium onset can be linked to relevant clinical and policy outcomes or important metrics, such as 30-day re-admission rate.

- The work group should also explore how the Age Friendly Health System-developed around the four "M's" of mobility, (what) matters, medication, and mentation-may aid in the identification of delirium occurrence.

- A work group should explore the concept of important drivers of change. The American Hospital Association, Institute for Healthcare Improvement, US federal/state entities, the Healthcare Quality Improvement Partnership (UK), and other international groups might be important thought partners, as well as potential collaborators, for future demonstration projects.

- Future work groups should establish a process to update standards annually; these activities should adopt recursive and iterative reviews that rapidly communicate new knowledge and areas of emerging consensus.
The literature for delirium prevention is more robust than that for delirium treatment. ${ }^{9}$ The application of awareness campaigns that promote interventions to prevent delirium will likely be the most effective near-term strategies to reduce delirium and its sequelae. These approaches can often be applied rapidly to influence clinicians, patients and their family members. ${ }^{13}$ The group reviewed case studies evaluating various efforts from different stakeholders on how best to educate medical and care services professionals.

\section{Educating the work force: Non-profit efforts}

The Hospital Elder Life Program (HELP, HospitalElderLifeProgram.org), created in 1993 with support from the NIH, Commonwealth Fund, and Retirement Research Foundation, was the earliest delirium prevention model of care. ${ }^{14}$ This program demonstrates that delirium could be prevented in some patients hospitalized on the general medical and surgical floors. Now, in partnership with the American Geriatrics Society, HELP is an international community of like-minded clinicians and volunteers dedicated to the prevention of delirium in vulnerable populations.

- Created website resources for the public, which are updated regularly

- Created multimedia training modules for workforce development, which are updated regularly

- Hosts annual meetings and interest group meetings throughout the year

- Established HELP Centers of Excellence in United States and Canada

- Instituted GoogleGroups to support HELP sites

The American Board of Internal Medicine developed a program entitled "Choosing Wisely" to promote discussion between clinicians and patients by helping patients choose the most effective care options. The effort includes an awareness module of delirium in dementia for care-providers. An enduring challenge, particularly in the dementia population, is that although delirium assessment may be performed, remedies are inconsistently applied and may ignore current best evidence.

In 2008, the Hartford Foundation, the American Academy of Nursing, and the Universities of Kansas, lowa, and Pennsylvania took on a project, the Geropsychiatric Nursing Collaborative to infuse geriatric nursing content into the nursing curriculum. In doing so they developed competencies and content.

- Developed teaching resources for faculty 
- Identified "Top three things needed"-a delirium checklist, tools, policy document

- Developed a document on delirium prevention.

This focus on delirium prevention has also expanded into the intensive care unit (ICU) setting. The Society of Critical Medicine (SCCM) has demonstrated that use of the $A B C D E F$ bundle $(A=$ Assessment and treatment of pain; $B=$ Both spontaneous awakening and breathing trials; $\mathrm{C}=$ Medication choice and de-escalation; $\mathrm{D}=$ Delirium screening and prevention; $\mathrm{E}=$ Early mobilization; and $\mathrm{F}=$ Family engagement) will reduce ICU days without delirium or coma by more than half. ${ }^{15}$ Use of this bundle shortens time on the ventilator in the ICU by $>2$ days, facilitates more patients going to home or rehab (vs a chronic care facility) and significantly reduces the rate of 30-day hospital readmission.

- Description of ABCDEF bundle (SCCM ICU liberation website)

- A link to the results of the Large-Scale outcome study ${ }^{16}$

Because the medical community frequently does not detect delirium and is often focused on other medical concerns, it often fails to provide education to patients and their families about delirium, including the most common risk factors for its occurrence and the negative short- and long-term outcomes that may occur. In response, the American Society of Anesthesiologists (ASA) started a multi-stakeholder initiative focused on post-operative delirium. This initiative includes the development of a website ${ }^{17}$ targeting patients and providers, grand rounds at CMS, and a proposal for the medical community in collaboration with The Joint Commission (TJC) to construct a protocol that will address mentation around surgery and potential delayed cognitive recovery after surgery and will better communicate this information to the public. AARP has partnered with the American Society of Anesthesiologists (ASA) and is working on infographics that will be disseminated through the American Hospital Association, the American Geriatrics Society, private hospital systems, and the lay press. The American College of Surgeons created Optimal Resources for Geriatric Surgery Standards and a Geriatric Surgery Verification Program in concert with a multi-stakeholder group with funding by The John A. Hartford Foundation. Thirty new surgical standards were designed to systematically improve surgical care and outcomes for the aging adult population including those related to postoperative delirium.

The American Delirium Society provides tools on their web site and delirium education cards. ${ }^{18}$

\section{SUMMARY ISSUES}

- Preventive methods can reduce the risk of delirium and are recommended in all delirium clinical guidelines. ${ }^{9,19}$ It is recommended that all healthcare professionals receive required training on delirium recognition and prevention. ${ }^{9,20}$ Education of the general public on delirium recognition and prevention is also critical.

- The work group should produce a Delirium Almanac of past, established, and future activities. The main idea would be to provide a catalog of activities with details and key contacts. The Delirium
Almanac should capture activities such as (but not limited to) the SCCM Pain, Agitation/Sedation, Delirium, Immobility, and Sleep Disruption in Adult Patients (PADIS) guidelines, the Scottish Intercollegiate Guidelines Network guidelines on delirium, the activities of Institute for Health Care Improvement (IHI), the Age-Friendly Movement, the efforts of American Hospital Association (AHA) and The Joint Commission (TJC), and major clinical databases and audits. $^{11}$

- The work group should also attempt to incorporate a Consumer Reports-style rating of different delirium awareness activities within the Delirium Almanac. Activities can be evaluated based on existing and available metrics such as audience size, time length of program, cost, and patient and clinician perceptions, and so on.

\section{Educating the work force: government efforts}

Several areas exist for potential collaboration with federal and regional governmental organizations. In the United States, the CMS has partnered with the Agency for Healthcare Research and Quality, the Centers for Disease Control and Prevention (CDC), Health Resources and Services Administration (HRSA), and others on quality improvement efforts to reduce all-cause harm. ${ }^{21}$ The U.S. Department of Veterans Affairs has also identified this as an important area and has a website for delirium that includes its risk factors, who should be screened, and information about prevention. The CDC has limited activities in this area. In addition, HRSA has a geriatrics workforce enhancement program (GWEP) to improve health outcomes in older adults. All GWEP grant recipients are required to educate healthcare clinicians on the care of older adults with mental health issues including delirium. The key component of this funding vehicle is a partnership requirement between academia, primary care, and community-based organizations. The ACL is the federal agency focused on increasing access to community supports for older Americans and people with disabilities across the lifespan. It has mentioned delirium in consumer-facing materials (eg, factsheets), which have covered the topics on aging such as opioid use in older adults and how states and communities can become dementia friendly. The UK and Australian governments have produced substantial policy initiatives concerning better standards of delirium care.

\section{SUMMARY ISSUES}

- Government programs need to address the challenge of how to better identify and prevent delirium, with language specifically tailored to clinicians and lay audiences.

- Government programs and accreditation agencies (eg, TJC [formerly the Joint Commission on Accreditation of Healthcare Organizations, or JCAHO]) should create incentives for delirium recognition and prevention. This means expanding the focus of delirium prevention monitoring strategies across different types of clinical settings to also include measurement/assessment of implementation of unique quality/process constructs.

- Certification requirements should be developed for healthcare professionals to recognize and prevent delirium. Awareness programs 
to educate clinicians about these requirements need to be formulated. Requirements for educational programs for delirium recognition and prevention have been developed recently by organizations like the American Delirium Society.

- Initiatives are needed to track outcomes, particularly negative ones most commonly associated with delirium.

- Success of these initiatives should also be evaluated. A discussion of how to define success is needed, including rates of delirium detection in practice and adherence to delirium prevention strategies.

\section{The situation on the ground and the current hard realities}

Currently, only a very small fraction $(\leq 3 \%)$ of delirium cases are diagnosed in hospital records. There are multiple factors contributing to this low level of reporting, and it is important to map out the care ecosystem as a first step toward developing a blueprint that is comprehensive and balanced so as to fulfill the intent of preventing delirium, and improving outcomes for patients who develop delirium. The blueprint development could be informed by lessons learned from the well-intentioned payment policy that attempted to address falls by including falls as one of the 14 quality indicators that hospital were already tracking.

Following this policy, patients age 65 and older were screened for fall risk, and in some hospitals, patients determined to be a potential fall risk were required to have bed rails up or bed/chair alarms enabled. In this circumstance, patients are effectively immobilized. This scenario transpired despite the lack of inclusion of falls screening in the State Operations Manual (SOM) that provides written guidance for the implementation of the Medicare Conditions of Participation for hospitals and facilities. Moreover, the use of restraints for the prevention of falls should NOT be considered a routine part of a falls prevention program and that here is no evidence that the use of physical restraint (including, but not limited to, raised side rails) will prevent or reduce falls. The SOM specifically mentions that raised side rails can pose a greater risk to patient safety if applied to a patient who attempts to climb over them. It is important to note that the need for an individualized patient assessment is emphasized, as is the least restrictive intervention, and the need to ensure a safe environment. This cautionary example of "falls in hospitals" highlights the need for awareness efforts to carefully define the outcome, such as "falls with major injury" and then to ensure consistent fidelity among the definition, the interpretation, and the desired outcome. In the case for "falls in hospital," the interpretation of the CMS ruling led to a cottage industry for suppliers of bed alarms.

The necessity of this approach can be further illustrated by the example of what could happen if delirium were included on a facility/hospital report card for public reporting (ie, a quality indicator). The result could be that facilities and their staff begin to employ unstandardized definitions, inconsistent use of standardized detection tools, and develop an order set that includes routine use of antipsychotics (which have not been shown to improve delirium outcomes). This approach could be even more counterproductive if event reporting is not coupled with clinical tools to enhance delirium detection and evidence-based management. Although policies are revised at regular intervals, it takes time to reverse the care practice.

The National Quality Forum was funded to write a playbook for delirium. One focus is to foster development of a single quality metric. There is a need for a quality metric focused on post-operative delirium detection. Other committees, including the United States Senate Special Committee on Aging, are also interested in delirium. The Patient Safety Movement took up delirium in 2019. The Choosing Wisely framework was discussed. The ABM Foundation (developer of Choosing Wisely) would enthusiastically take this approach with delirium.

\section{SUMMARY ISSUES}

- Highly precise messaging-language and tools regarding delirium awareness are needed. These can be a framework, a protocol, and/or a hybrid multifaceted media approach. This will also require the work group to develop clear definitions, anticipate scenarios where efforts could be misinterpreted, and define best practices to measure outcomes and evaluate differences.

- There is a need to build systems to prevent or offset unintended consequences:

- Delirium is a patient behavior and highlights the fact that this behavior may be difficult for clinicians and family members to understand. For example, if a patient wakes up with chest pain, both the clinician and the patient/family member will assume an underlying heart condition is present. However, clear assumptions of an underlying brain condition are rarely made with respect to an episode of delirium. Clinicians often intuitively consider use of a medication (eg, an antipsychotic) to treat delirium, despite this not being a recommended first-line treatment for delirium. ${ }^{9}$ These medications are easy to administer and the pharmacological rationale for these practices have been perpetuated. Yet, the problem of using medications solely to treat delirium requires a major educational and awareness effort particularly given the weak evidence supporting current pharmacological interventions. As the pathophysiological mechanisms remain under investigation, non-pharmacological interventions have demonstrated far better evidence supporting their use for delirium reduction. As future work groups focus on this issue, it is worth noting that with angina, the goal is preserving cardiac function. In the case of delirium, the goal should be focused on how best to preserve brain function.

- Global efforts should include a resource list of quality initiatives that can be implemented

\section{2 | Informing the general public}

AARP and its Global Council on Brain Health (GCBH) have taken an important lead to drive awareness of brain health issues and brain function preservation. To expand these efforts, AARP and the American Society of Anesthesiologists brought experts from around the world to participate in a forum to discuss delirium in 2018. Then 
in 2019, AARP convened the GCBH to address delirium from the patient's perspective. The GCBH identifies brain health topics of interest to adults, pulls in independent experts, and works toward a focused consensus on the state of the science. The issues are distilled as clearly as possible for the general public. Information about what families and caregivers can do (ie, tips for recognizing and preventing delirium in a loved one) are highlighted, and current gaps in knowledge are summarized. Once such a report is finalized, AARP makes the information available to their membership of 38 million people and to others around the world through the GCBH's global partnerships.

AARP partners with other trusted providers of health information to consumers such as the $\mathrm{NIH}, \mathrm{CDC}$, and the $\mathrm{ACL}$ and others to communicate a unified message about delirium to the public (as well to clinicians and researchers). AARP has a strong partnership with anesthesiologists but has only begun the journey to educate older adults and their families about delirium. Work remains to be done to develop a unified message, but AARP anticipates releasing the GCBH delirium report in Spring 2020.

The GCBH is most effective when statements to the general public are simple and clear, and the solutions are easy to adapt and deliver. Solutions should generally not be driven by the latest science, but instead by information for which firm consensus exists among experts.

\subsection{1 | General public: A caregiver-centric perspective}

Caregivers usually have an intimate role in delirium recognition and reduction. This role generally becomes active during hospitalization and may become long-term after discharge. Most healthcare systems could vastly improve their performance in reducing delirium when caregiver-centric approaches are used. Public health campaigns must link awareness efforts with the provision of individually tailored solutions. For example, in the United States, Medicare usually stops paying for services 30 days after hospital discharge; very few services are available to help reduce delirium and its cognitive effects on day 31 despite the fact that older adults with delirium may have deliriumrelated symptoms for weeks.

Some Canadian hospitals have engaged in a campaign called "this is not my mom." 22 If a family says, in effect, "this is not my mom," that is, my mom does not usually behave this way, the hospital must act. Similarly, when healthcare professionals encounter a patient and "you can't get through to them," they have to ask, "Is this new"? Using these approaches, delirium recognition rates improved from $<5 \%$ to $>15 \%$ after only 5 weeks. Since the recognition rate tends to decrease a few months after initiation of new programs, education programs regarding these initiatives are conducted annually to reinforce that healthcare professionals need to ask these delirium screening questions.

Caregivers benefit most from brief recommendations due to limited time and a heavy workload. Social media is an important communication channel through which many children and grandchildren may provide supplementary, virtual caregiving. More patient-level and caregiver-level focus group assessment is needed. For example, many patients/caregivers seek advice about how to determine when cognitive changes are normal versus not normal. However, knowing the baseline cognitive function of the person is crucial to accurately answer this question. ${ }^{23}$ The ACL has funded some important initiatives around this topic. One example is the Eldercare Locator, which connects family caregivers, families, and older adults with resources in their community. Surveillance systems will need to be able to identify and capture those events and symptoms that may provide important background information. ${ }^{23}$ Such information might inform clinicians about what led a patient to the hospital and whether a cognitive change is acute or chronic

One of the most effective ways for clinicians to understand these issues and for patients and their family members to convey suspected delirium to their clinicians is to formalize the approach by which patients, family members, and their clinicians tell stories. In this way, patients and family members can convey a message that is highly important. The idea of witnesses who experienced delirium is a powerful means by which to communicate information to individuals who are at high risk for experiencing a similar situation.

\section{SUMMARY ISSUES}

- There is a clear need to develop both integrated public awareness and engagement campaigns surrounding delirium. Future work groups will need to address how the effectiveness of these campaigns can be evaluated.

- Messaging: A catchy, easy to understand type of message that sounds powerful, pithy, and promotes public awareness is needed.

- Cross-cultural perspectives are critical for any worldwide awareness effort. Social media is a powerful global messaging tool.

- Future work groups should develop demonstration projects around:

- The use of electronic medical data (eg, case notes, antipsychotic use, surveys of clinician beliefs, and practices) to inform the development of delirium education/quality improvement programs.

- The integration and enhancement of patient, family, and caregiver communication.

- Boosting public engagement: A hub website should be developed to include detailed information about HELP (HospitalElderLifeProgram.org), the ABCDEF bundle, and the "think delirium" initiative. ${ }^{24}$

- The development of a series of virtual grand rounds, would provide a forum in which case stories may be used to educate both caregivers and healthcare professionals. The stories may help people understand post-surgical outcomes before a surgical intervention occurs in an effort to provide patients and their families with better tools to navigate the healthcare system.

\section{7 | ITEM 3: NEUROBIOLOGICAL BASIS OF DELIRIUM}

The neurobiological basis of delirium is not well understood. This gap in our knowledge is reflected in our description of delirium guided mainly 
by the clinical features detailed in the DSM- 5 criteria. However, there are opportunities to build an understanding of delirium biology on the dual foundations of these clinical symptoms and our existing knowledge of the chronic vulnerabilities and acute precipitants that trigger delirium. The key feature of delirium is an alteration in attention and awareness. This can present as various states-hypoactive, hyperactive, or a mixed presentation due to a fluctuation between these two states. This fluctuation between these various states of wakefulness and arousal implicates the reticular activating system (RAS). The RAS is a group of neuronal populations that arises in various regions of the midbrain and projects to central thalamic nuclei to control cortical states of arousal. These populations (such as the cholinergic pedunculopontine nucleus) are tonically active during alert wakefulness, drive high frequency oscillations including gamma waves $(30-80 \mathrm{~Hz})$, and shift to more phasic firing, which is the underpinning of the slow wave delta (1-4 Hz) activity prominent during sleep, anesthesia, and lower states of consciousness such as delirium. A depolarized active mode is required in the central thalamic nuclei for attentive states and performance of tasks that need persistent frontal cortical activity, such as attention and working memory. ${ }^{25}$ Conversely, phasic activity in these thalamic neurons drives EEG synchronization and slow-wave activity, resulting in failure to support brain functions that depend on high arousal state.

These shifting arousal states are typically entrained to a circadian rhythm by the suprachiasmatic nucleus of the hypothalamus. Degeneration in this region in dementia, and to a lesser degree, aging, ${ }^{26}$ loosens this entrainment and makes transitions between these arousal states unpredictable and often leads to problematic behavior. One such problematic behavior may be the commonly observed, but poorly characterized, syndrome known as "sundowning." During sundowning, patients with dementia become even more noticeably disorientated to time and place in the evening, which can lead to increased agitation and wandering.

Limbic regions such as the hippocampus, amygdala, and the frontal and parietal cortices also significantly influence activity of the RAS. Significant degeneration of the hippocampus during dementia, as well as significant amygdala output during stress, can significantly disrupt the ability of the RAS to mediate appropriate cortical arousal. Structural and functional neuroimaging studies have implicated the HPC, thalamus, RAS, and connectivity between these regions as potential key players in delirium pathophysiology. ${ }^{27,28}$ Thus, age and neurodegenerative disease-associated changes in circadian, hippocampus, and emotional processing in the amygdala may significantly impinge on the typical maintenance of appropriate brain arousal states. A detailed study of the functional connectivity between regions before or during delirium would allow for a deeper understanding of networks in delirium, which could eventually surpass our current understanding of similar pathways in dementia.

Thus, the research on the neurobiology of delirium needs to significantly extend its horizon by cultivating multiple approaches. An important starting point would be to further explore the biological pathways that are shared by both delirium and dementia. One existing point of entry that has been explored is that of neuroinflammation.
Inflammatory "priming" of microglia or other cell populations in the aged or degenerating brain leaves these brains vulnerable, such that acute stressors such as infection, injury, or surgery may now disproportionately affect those with pre-existing neurodegenerative pathology. ${ }^{29}$ Dissecting the different levels at which the degenerating brain is vulnerable to acute stressors will be important in identifying mechanisms by which these stressors might trigger delirium. Likewise, understanding the pathways by which particular vulnerabilities predict the negative long-term outcomes of delirium will be key to identifying at risk patient groups and to developing targeted therapies. It is important to examine differential effects of delirium between populations that have subjective cognitive impairment, mild cognitive impairment, and dementia. Mouse model and human data show that progressive underlying degeneration, or decline, progressively increase risk for delirium. ${ }^{30}$ It is now essential to explore ways to predict which specific brain regions may become dysregulated. The research agenda should also explore unmasking the vulnerability to episodes of delirium by developing stress tests to examine what regions or functions of the brain are, or are not, working at capacity to remain above thresholds for preserving function.

\section{1 | Obstacles to progress}

The number of neuroscience researchers focused on delirium is relatively small; its growth should be a major goal. One of the barriers is growing a community of researchers interested in this area. The basic neuroscience community is rather large. Therefore, a key challenge will be to attract those researchers active in the neuroscience of arousal, attention, circadian rhythm, stress, inflammation, blood-brain barrier mechanisms, and brain metabolism, to the fascinating problem of delirium. Upstream mechanisms may also emerge from studies in anesthesia. For example, one form of delirium emerges when sub-anesthetic doses are administered; this situation could also be modeled in animals to more deeply understand delirium pathophysiology. Efforts to attract and collaborate with researchers across this breadth of fields in neuroscience can be pursued at an institutional level as well as through courting researchers from outside the delirium field for satellite symposia as recently trialed by the European Delirium Association. ${ }^{31}$

The establishment of a research resource core focused on delirium would include human studies, a biospecimen bank, neuroimaging, and physiology studies. In the midst of developing a resource of animal model studies, there is a critical need to integrate the communication of research findings among neuroscientists doing work relevant to delirium but as yet unaware of potential relevance to delirium, as listed above.

Work groups will need to challenge the popular misconception that delirium may be too complex to systematically study. For the outsider, delirium has a multifactorial etiology, has multiple phenotypes, is characterized by a fluctuating rather than steady state, and is a complex system with multiple subsystems interacting, compensating, and being modulated by positive or negative stimuli. Although the transient nature of delirium makes an accurate diagnosis a challenge in clinical 
settings, it presents a unique opportunity for basic neuroscience research, since those transient states can be experimentally induced and controlled. Despite the complexity of delirium, the work group will need to carefully consider more reductionist animal models that allow causality to be studied. In this way, using model systems will allow specific hypotheses to be tested and yield predictions that can be examined in more complex models and in people.

For delirium, the field will need to accept the possibilities for multiple, differing routes to brain dysfunction. This means that those that prefer to either lump or split will need to embrace an ecumenical thinking of all etiological pathways so that we recognize complex interactions from multiple upstream and downstream events.

\section{SUMMARY ISSUES}

- The work group will need to evaluate and establish the goals for development of new model systems and also prioritize studying, at greater neuroscientific depth and breadth, those model systems already in use.

- Recognize the need to prioritize the development of effective treatment strategies, particularly pathophysiological-based targeted treatments.

- Work group should make specific recommendations for funding of key research questions and infrastructure needs. How much is needed in the next 5 years? What is needed to increase recognition across the broader scientific community? How to gain interest from other basic scientists in the relevant areas: Satellite symposia, additional NIA workshops, or summits on delirium with targeted invitations?

\section{I ITEM 4: BURDEN DISCUSSION}

Developing credible estimates of the public health and economic burden of delirium will be a key task for the IDID campaign. In an era of decreasing availability to healthcare resources and increasing demand, there is a critical need to determine the relative value of evidence-based interventions, services, and programs for identifying, treating, and generating awareness about delirium. Although it is well-established that delirium is associated with increased resource utilization and costs of care, ${ }^{32}$ there is an urgent need to develop detailed population-based data regarding the incidence, duration, and costs of delirium across different ages, clinical settings, geography, and ethnicities.

The most pressing challenge for work groups will be to devise a research strategy that can better address the needs of public policy experts who are focused on delirium. For example, reliable and valid cost estimates for the burden of delirium experienced by patients and their caregivers are needed. A working group should explore how to establish a common methodology for cost-of-illness studies for delirium.

A standardization of terminology will facilitate comparison across studies and allow pooling and re-use of results from subsequent, longer-term evaluation studies. Efforts should be made to improve the measurement and valuation of the informal care invariably delivered to patients with delirium or post-delirium sequelae provided by family caregivers. This is an important avenue for methodological development given the substantial variation in both terminology and methodology. ${ }^{33}$ Finally, a future work group should explore those opportunities to meld patient care and research activities. As precision medicine initiatives continue to expand, there will be an important need to pinpoint individual variability in resource utilization and cash flow for each stakeholder/payer along the trajectory of a delirium episode.

The discovery and development of cost-effective interventions for delirium will need to be a global effort that is informed by rigorous global clinical trials. The evidence generated must fulfill not only regulatory requirements but also inform private and public budget holders responsible for allocating funding for existing and new interventions. Future work groups should identify opportunities to align evidence requirements among regulators, payers, and other private and public stakeholders. Health policy formulators in countries outside the United States, particularly among countries with single-payer systems and those considered resource-poor, will likely have different perspectives on how new delirium-reducing interventions are evaluated and the evidence required to define an intervention as being necessary and sufficient. Published recommendations, such as the Second Panel on Cost-Effectiveness in Health and Medicine, emphasize a structured framework for presenting results from economic evaluations. ${ }^{34}$ The work group should try to incorporate the panel's recommendation for reporting two reference cases: A health care sector and a societal perspective and include all consequences irrespective of budget holders.

Health economic evaluations traditionally use Quality Adjusted Life Years (QALYs) to quantify net health benefits of a new medicine or nonpharmacologic intervention. QALYs provide a means to develop value for new interventions in which net costs are seen in relation to the net health benefits conveyed by the new intervention. Although this single currency construct, often applied across multiple diseases, is attractive from a theoretical perspective, deriving credible estimates remains a practical challenge, particularly among disorders affecting cognitive function. Future work groups should seek alternatives, such as value frameworks (taken from oncology), in which the application of a scoring algorithm quantifies net health benefit of a new intervention in terms of its clinical effectiveness against any potential harms. ${ }^{35}$

\section{SUMMARY ISSUES}

- The public health and economic burden of delirium needs to be fully characterized to appreciate the true impact of this condition. Specific health economic modeling work should focus on developing microsimulation methods that can estimate individual disability adjusted life-years that is embedded in real-world clinical care and hospital environments.

- Studies need to characterize utilization patterns that consider culturally, ethnically, and geographically disparate and/or underserved populations. 
- The downstream costs of delirium include many factors such as direct health care and caregiving cost, as well as a myriad of indirect costs due to new disability and/or accelerated cognitive decline, and that are potentially related to or a consequence of delirium. The impact of delirium on lifespan and post-hospital survivorship requires:

- Research on how and to what extent the downstream costs of delirium including direct health care and caregiving costs, indirect costs due to new disability, and/or accelerated cognitive decline are potentially related to delirium, the impact of delirium on lifespan, and post-hospital survivorship.

- Forecasting methods to improve the estimation of new delirium cases from different clinical settings in the short, medium, and long term.

- There needs to be international consensus on the definition of the reference standard or standards that define a case or episode of delirium.

\section{9 | ITEM 5: DISCUSSION OF AWARENESS RAISING}

The fragmentation of clinical care remains a difficult problem and is exacerbated among patients with delirium. For example, the anesthesiologist who evaluates a patient undergoing a major surgical procedure pre-operatively is rarely the same as the anesthesiologist who provides care in the operating room. Nor are the clinicians who cares for the patient in the ICU the same as those who manage the care transition to the floor. Some individuals with delirium, particularly those who survive a critical illness, may be routinely discharged from the hospital with unrecognized delirium. The post-hospital expectations of these patients and their families need to be managed better. Specifically, the marketing of medical care needs to shift away from simply touting technology. Instead, the emphasis should be making the individual patient better, faster. With this new emphasis, symptom-based care will focus on improving hospital survivorship and brain health. There is a pressing need to educate and convince medical care consumers that some health centers will take better care of you and your brain than others. This is included in the concept of the age-friendly health system.

The task of building patient demand for better care is important and has to be a win-win for patients, families, and clinicians. There are two key historical precedents for rapid implementation. The application of anesthesia quickly gained adoption across the world in a matter of months (before the internet) and handwashing was recognized by consumers as a means to prevent infection. Although, many individuals confronted with a middle-of-the-night chest pain would know how to respond, there are no clearly defined actions plans for how to respond to an acutely confused individual. There is a pressing need to educate the public about the differences between dementia and delirium, and that among other differences in management, in the latter, your brain may be in acute danger.

A number of key stakeholders will be critical to developing an awareness campaign. These include hospital physicians (hospitalists),
TAB LE 1 Organizations/programs with a focus on delirium and/or brain health

\begin{tabular}{|c|c|}
\hline $\begin{array}{l}\text { AARP and the Global Council on } \\
\text { Brain Health }\end{array}$ & $\begin{array}{l}\text { Administration for Community } \\
\text { Living } \\
\text { Alzheimer's Association }\end{array}$ \\
\hline $\begin{array}{l}\text { Alzheimer's Drug Development } \\
\text { Foundation } \\
\text { American Association of Critical } \\
\text { Care Nurses }\end{array}$ & $\begin{array}{l}\text { American Federation for Aging } \\
\text { Research } \\
\text { American Association for } \\
\text { Geriatric Psychiatry } \\
\text { American College of Clinical } \\
\text { Pharmacists }\end{array}$ \\
\hline $\begin{array}{l}\text { American College of Emergency } \\
\text { Physicians }\end{array}$ & American College of Physicians \\
\hline American College of Surgeons & $\begin{array}{l}\text { American Congress of } \\
\text { Rehabilitation Medicine }\end{array}$ \\
\hline American Delirium Society & American Geriatrics Society \\
\hline American Hospital Association & American Nurses Association \\
\hline $\begin{array}{l}\text { American Society of } \\
\text { Anesthesiologists } \\
\text { American Thoracic Society } \\
\text { Centers for Medicare \& } \\
\text { Medicaid Services }\end{array}$ & Australian Delirium Association \\
\hline European Delirium Association & $\begin{array}{l}\text { The Gerontological Society of } \\
\text { America }\end{array}$ \\
\hline $\begin{array}{l}\text { HCSRN/Claude D. Pepper Older } \\
\text { Americans } \\
\text { Independence Centers } \\
\text { Institute for Healthcare } \\
\text { Improvement } \\
\text { Health Resources and Services } \\
\text { Administration }\end{array}$ & $\begin{array}{l}\text { John A. Hartford Foundation } \\
\text { British Geriatrics Society } \\
\text { International Delirium } \\
\text { (iDelirium) }\end{array}$ \\
\hline $\begin{array}{l}\text { Hospital Elder Life Program } \\
\text { (HELP) }\end{array}$ & $\begin{array}{l}\text { Network for Investigation of } \\
\text { Delirium: Unifying Scientists }\end{array}$ \\
\hline $\begin{array}{l}\text { National Institute on Aging } \\
\text { National Heart, Lung and Blood } \\
\text { Institute }\end{array}$ & $\begin{array}{l}\text { Retirement Research } \\
\text { Foundation }\end{array}$ \\
\hline $\begin{array}{l}\text { Nurses Improving Care for } \\
\text { Healthsystem Elders (NICHE) }\end{array}$ & $\begin{array}{l}\text { Society of General Internal } \\
\text { Medicine }\end{array}$ \\
\hline Society of Critical Care Medicine & Scottish Delirium Association \\
\hline Society of Hospital Medicine & WestHealth Foundation \\
\hline
\end{tabular}

surgeons, anesthesiologists, geriatricians/associated subspecialties, pharmacists, nurses, occupational therapists, primary care providers, and health care assistants. Although not an exhaustive list, several societies and associations should be represented (Table 1).

Currently, there are several communication channels open to help raise delirium awareness, but the message remains unfocused and unclear. The result: Message penetrance is soft. Direct care for patients with delirium is mostly delivered by nurses. However, important structural deficits surrounding the education and training delivered to nurses regarding delirium recognition and prevention exist. The didactic training most nurses receive in delirium is miniscule ${ }^{36}$ compared to the estimated $20 \%$ of their time that ultimately is devoted in their professional careers to patients with delirium. An important case history 
describes the experience of a 52-year-old individual who had delirium for a month. The patient later reported that 1 day a nurse held her hand, looked in her eye, and said she would be safe: That was the only point when the patient said that she truly felt safe.

A key aim for any awareness campaign is to humanize the delirium condition and improve the graduate and post-graduate training deficit such that nurses feel they are a critical member of an interdisciplinary care team. To implement this activity, a work group will need to carefully survey the landscape and identify where duplication of delirium-reduction efforts exists. Part of the work group efforts might examine how and why many nurses are frustrated with their ability to recognize and reduce delirium symptoms in their patients. Training modules should go beyond the traditional constructs and also focus on the limited role of pharmacologic management, and instead empower nurses to deliver effective nonpharmacologic prevention and management strategies.

\subsection{Key to have public pulling as much as we are pushing}

Public education requires clear and concise communication given the wide variation of awareness of delirium that exists among patients and their families. A work group will need to manage the expectations of patients and families, and balance these against the current delirium and risk-reduction practices that have been implemented in the health system where they work. Public engagement and representation in the work group will be important, along with the participation of consumer health advocacy groups.

A future work group will need to hone the message. This might involve creating smart phone apps that enable diagnostic screening at the home, accelerometers, or other tools to create alerts to caregivers. Collaboration with Meals on Wheels and other home-based delivery services may be useful where training is provided so that the delivery person can "ask one question a day."

Future work will need to carefully consider the process of global health messaging and how informing public opinion may lead to unintended outcomes. The power of stories is critical, such as having a celebrity who was affected either personally or via a loved one, is a great start. However, the effort must start with a collection of people who are willing to tell their story. "This is not my mom" and "brain fog" are campaigns that have worked, but better to let the public develop the messages.

\section{SUMMARY ISSUES}

- A key aim for a public awareness campaign will be to humanize delirium and to improve the quality of graduate and post-graduate training deficits so that bedside nurses are empowered and feel they are an equal part of the care team.

- Healthcare professionals may be a potential barrier to a public awareness campaign, particularly if they are not familiar with screening/assessment tools. Awareness campaigns should be cali- brated so that healthcare providers and systems remain engaged in ongoing continuing education to balance awareness efforts equally among professionals and the public.

\section{0 | ITEM 6: DISCUSSION OF WHAT WOULD BE NEEDED TO ESTABLISH A FULL-SCALE PUBLIC POLICY AND PUBLIC HEALTH CAMPAIGN TO DEFEAT DELIRIUM}

The work group focused on the question of how to build a successful coalition to improve categorically the care of delirium. There are several models that can be instructive, recognizing that each policy problem has its own unique features. In addition, it is important to recognize that opportunities may be heavily influenced by the ever-shifting political climate; careful navigation and strategic planning are required. It is noteworthy that the shifting political climate includes not only elected representatives, but also the medical and scientific community. If politics is the art of possible, then political artists must understand what is possible but seems presently to be impossible.

One notable case example was the launch and development of the U.S. national program on aging and AD. The program came into existence in 1978 as a means to address the public health epidemic of chronic disease associated brain aging. At the time, chronic brain diseases such as $A D$ were stigmatized both in medical and scientific circles and considered as implausible to treat. Yet, in just over 10 years the first treatment for $A D$ was approved by U.S. Food and Drug Administration (FDA).

Perhaps the most important case study for the development of a delirium public health campaign is the history of the National Alzheimer's Project Act. The current effort on prevention started in 2007 with the creation of the Leon Thal Symposium and the Alzheimer's Study Group (a U.S. Congressional mandated blue-ribbon work group) to prepare a report to solve the growing problem of AD. These work groups, established independently, eventually came to work together and bring about the initial framework for a public health agenda to solve the dementia crisis. The Alzheimer's Association put together another work group in 2009 , which estimated that it would require a sustained investment of approximately $\$ 2$ billion per year over a 10-year period to meaningfully reduce the disease's burden.

The challenge for developing a public policy initiative focused on delirium will need to determine how to target the issues and effectively convey the scope of the problem posed by delirium. Future work groups will need to isolate those key parameters around which people can build a public health story that will lead to the formation of a natural coalition. One part of this effort may need to expand on the work led by AARP, to drive greater awareness and to eliminate stigma and stimulate focused interest from within the research community as well as the larger public. Another critical component will be to devise strategies and options to build public support. Many disease-advocacy groups were created in this manner, and it may be possible that the NIH can help play an important role again to galvanize the area in the future: Advocacy groups and spokespeople will be needed. 
A special work group should be formed to develop a public policy business plan that will define the problem, the relevant stakeholders' perspectives, and how a "delirium public health campaign" will be understood by everyday people and voters. This work group will need to interface with many disciplines and expertise to craft the message of $a$ tractable problem with a viable solution. This work group will also need to specify the size and timeline for a credible management group to deliver results, the major/key initiatives to start, and finally to establish/socialize the "big ask" necessary to reduce the societal burden due to delirium.

\section{SUMMARY ISSUES}

- Design a strategy to support and staff a dedicated public policy team that manages the stakeholders' perspectives and develops key/central messaging.

- Define what "viable solutions" might mean for delirium control.

- Author a compelling "delirium public health" story.

- Invite allied advocacy groups to participate in this effort and leverage resources and messaging.

- Develop a "big ask" that will spur investment to mitigate the burdens due to delirium.

\section{ACKNOWLEDGMENTS}

AARP, Vradenberg Foundation, PAD2020, Wake Forest University for their financial and logistical support for the production of this meeting and report; Dr. Molly Wagster, for her attendance at the March 16, 2019 meeting in Washington, D.C., and for her careful comments of the historical programmatic citations in the manuscript; and Dr. Sophia Wang for her careful review of and commentary on the draft manuscript.

\section{REFERENCES}

1. Hanney SR, Castle-Clarke S, Grant J, et al. How long does biomedical research take? Studying the time taken between biomedical and health research and its translation into products, policy, and practice. Health Res Policy Syst. 2015;13(1):1.

2. Siddiqi N, Harrison JK, Clegg A, et al. Interventions for preventing delirium in hospitalised non-ICU patients. Cochrane Database Syst Rev. 2016;3:CD005563.

3. EW, Kaplan PW, Latronico N, et al. SAJCOWMDJWARCBTPCJDMSE. Updated nomenclature of delirium and acute encephalopathy. Intensive Care Med. In press.

4. Jack CR, Bennett DA, Blennow K, et al. NIA-AA Research Framework: toward a biological definition of Alzheimer's disease. Alzheimers Dement. 2018;14(4):535-562.

5. Neufeld KJ, Nelliot A, Inouye SK, et al. Delirium diagnosis methodology used in research: a survey-based study. Am J Geriatr Psychiatry. 2014;22(12):1513-1521.

6. Maldonado JR. Delirium pathophysiology: an updated hypothesis of the etiology of acute brain failure. Int J Geriatr Psychiatry. 2018;33(11):1428-1457.

7. Oh ES, Akeju O, Avidan MS, et al. A roadmap to advance delirium research: Recommendations from the NIDUS Scientific Think Tank. Alzheimer's Dement. 2020;16(5):726-733.

8. Evered L, Silbert B, Knopman DS, et al. Recommendations for the nomenclature of cognitive change associated with anaesthesia and surgery-2018. Anesthesiology. 2018;129(5):872-879.
9. Scottish Intercollegiate Guidelines Network (SIGN). Risk reduction and management of delirium. Edinburgh: SIGN; 2019. Healthcare Improvement Scotland. SIGN Evidence-based clinical guidelines Web site. https://www.sign.ac.uk/assets/sign157.pdf. Published 2019. Updated March 2019. Accessed January 21, 2020.

10. Tieges Z, Evans JJ, Neufeld KJ, MacLullich AMJ. The neuropsychology of delirium: advancing the science of delirium assessment. Int J Geriatr Psychiatry. 2018;33(11):1501-1511.

11. National Hip Fracture Database (NHFD) annual report 2018. Royal College of Physicians. https://www.rcplondon.ac.uk/projects/outputs/ national-hip-fracture-database-nhfd-annual-report-2018. Published 2018. Accessed February 2, 2020.

12. Faculty ANDCWGPCa. The American Geriatrics Society/National Institute on Aging Bedside-to-Bench Conference: research Agenda on Delirium in Older Adults. J Am Geriatr Soc. 2015;63(5):843-852.

13. Health ColSNCitDoHCtItNs. Integrating Social Care into the Delivery of Health Care: Moving Upstream to Improve the Nation's Health. Washington, D.C.: The National Academies of Sciences Engineering Medicine; 2019.

14. Inouye SK, Bogardus ST, Charpentier PA, et al. A multicomponent intervention to prevent delirium in hospitalized older patients. $\mathrm{N}$ Engl J Med. 1999;340(9):669-676.

15. Pun BT, Balas MC, Barnes-Daly MA, et al. Caring for critically ill patients with the ABCDEF bundle: results of the ICU liberation collaborative in over 15,000 adults. Crit Care Med. 2019;47(1):3-14.

16. Pun BT, Gordon SM, Peterson JF, et al. Large-scale implementation of sedation and delirium monitoring in the intensive care unit: a report from two medical centers. Crit Care Med. 2005;33(6):11991205.

17. Perioperative Brain Health Initiative. Amereican Society of Anesthesiologists. https://www.asahq.org/brainhealthinitiative. Published 2020. Accessed Janaury 17, 2020.

18. Medical Professional: Tools. American Delirium Society. https://amer icandeliriumsociety.org/resources/tools. Published 2019. Accessed January 17, 2020.

19. Devlin JW, Skrobik Y, Gélinas C, et al. Clinical practice guidelines for the prevention and management of pain, agitation/sedation, delirium, immobility, and sleep disruption in adult patients in the ICU. Crit Care Med. 2018;46(9):e825-e873.

20. Improvement IfHC. Initiatives Age Friendly Health Systems. Institute for Health Care Improvement. http://www.ihi.org/Engage/Initiatives/ Age-Friendly-Health-Systems/Pages/default.aspx. Published 2020. Accessed.

21. Plan CfMaMSCA-SQS. National Strategy for Quality Improvement in Health Care. Agency for Healthcare Research and Quality. https://www.ahrq.gov/workingforquality/about/agency-specific-quali ty-strategic-plans/nqs3.html. Published 2016. Accessed January 17, 2020, 2020.

22. Delirium Prevention and Care with Older Adults. Canadian Coalition for Seniors' Mental Health. Published 2016. Accessed January 20, 2020.

23. Eldercare Locator. U.S. Department of Health and Human Services, Administration for Community Living. https://eldercare.acl.gov/ Public/Index.aspx. Published 2018. Accessed January 20, 2020.

24. THINK Delirium. Canterbury District Health Board. https://edu.cdhb. health.nz/Hospitals-Services/Health-Professionals/think-delirium/ Pages/default.aspx. Published 2016. Updated August 2016. Accessed January 20, 2020.

25. Acsády L. The thalamic paradox. Nat Neurosci. 2017;20(7):901-902.

26. Witting W, Kwa IH, Eikelenboom P, Mirmiran M, Swaab DF. Alterations in the circadian rest-activity rhythm in aging and Alzheimer's disease. Biol Psychiatry. 1990;27(6):563-572.

27. Choi SH, Lee H, Chung TS, et al. Neural network functional connectivity during and after an episode of delirium. Am J Psychiatry. 2012;169(5):498-507. 
28. Cavallari M, Dai W, Guttmann CR, et al. Neural substrates of vulnerability to postsurgical delirium as revealed by presurgical diffusion MRI. Brain. 2016;139(Pt 4):1282-1294.

29. Cunningham C, Campion S, Lunnon K, et al. Systemic inflammation induces acute behavioral and cognitive changes and accelerates neurodegenerative disease. Biol Psychiatry. 2009;65(4):304-312.

30. Davis DHJ, Skelly DT, Murray C, et al. Worsening cognitive impairment and neurodegenerative pathology progressively increase risk for delirium. Am J Geriatr Psychiatry. 2015;23(4):403-415.

31. Pre-Meeting Pathophysiology of Delirium Satellite. European Delirium Association. http://www.europeandeliriumassociation.org/news/ pre-meeting-pathophysiology-of-delirium-satellite\#. Published 2019. Accessed January 20, 2020.

32. Pezzullo L, Streatfeild J, Hickson J, Teodorczuk A, Agar MR, Caplan GA. Economic impact of delirium in Australia: a cost of illness study. BMJ Open. 2019;9(9):e027514.

33. Wimo A, Gustavsson A, Jönsson L, Winblad B, Hsu MA, Gannon B. Application of Resource Utilization in Dementia (RUD) instrument in a global setting. Alzheimers Dement. 2013;9(4):429-435. e417.
34. Sanders GD, Neumann PJ, Basu A, et al. Recommendations for conduct, methodological practices, and reporting of cost-effectiveness analyses: second Panel on cost-effectiveness in health and medicine. JAMA. 2016;316(10):1093-1103.

35. Schnipper LE, Davidson NE, Wollins DS, et al. American society of clinical oncology statement: a conceptual framework to assess the value of cancer treatment options. J Clin Oncol. 2015;33(23):2563-2577.

36. Copeland C, Barron DT. Delirium: an essential component in undergraduate training? Nurse Educ Today. 2020;85:104211.

How to cite this article: Khachaturian AS, Hayden KM, Devlin JW, et al. International drive to illuminate delirium: A developing public health blueprint for action. Alzheimer's Dement. 2020;16:711-725. https://doi.org/10.1002/alz.12075 